\title{
Use of relative CT values to evaluate the invasiveness of pulmonary subsolid nodules in patients with emphysema
}

\author{
Bo-Wei Zhang ${ }^{1 \#}$, Yu Zhang ${ }^{2 \#}$, Jian-Ding Ye $^{2}$, Jin-Wei Qiang ${ }^{1}$ \\ ${ }^{1}$ Department of Radiology, Jinshan Hospital \& Shanghai Medical College, Fudan University, Shanghai, China; ${ }^{2}$ Department of Radiology, Shanghai \\ Chest Hospital, Shanghai Jiao Tong University, Shanghai, China \\ "These authors contributed equally to this work. \\ Correspondence to: Jin-Wei Qiang. Department of Radiology, Jinshan Hospital \& Shanghai Medical College, Fudan University, No. 1508 Longhang \\ Road, Shanghai 201508, China. Email: dr.jinweiqiang@163.com; Jian-Ding Ye. Department of Radiology, Shanghai Chest Hospital, Shanghai Jiao \\ Tong University, No. 241 West Huaihai Road, Shanghai 200030, China. Email: yejianding@126.com.
}

Background: Lung cancer is a major cause of death, and adenocarcinoma is the most common histologic subtype. Precise diagnosis and treatment of invasive adenocarcinoma (IAC) can substantially improve the survival of patients. However, early-stage adenocarcinomas frequently appear as subsolid nodules (SSN) on computed tomography (CT), and the optimal cut-off CT value for differentiating the invasiveness of SSNs in emphysematous patients is unclear.

Methods: High-resolution CT targeted scans of 187 pulmonary SSNs in 175 patients with emphysema as confirmed by surgery and histology were retrospectively reviewed. The mean CT value, the relative CT (rCT) values of 1 (nodule CT value - lung CT value), and 2 (nodule CT value/lung CT value), and the size of the SSNs were measured and calculated. The differentiating performance of the CT values between pre-invasive and invasive tumors was evaluated using a receiver operating characteristic (ROC) curve.

Results: Significant differences were found in the rCT values of 1 and 2 among pure ground-glass nodules (GGNs) with different levels of invasiveness, in the rCT values of 1 and 2 for the ground-glass component (GGC) and the mean CT value of the solid component (SC) of part-solid nodules (PSNs) between minimally invasive adenocarcinoma (MIA) and IAC (all $\mathrm{P}<<0.05$ ). The size was significantly different among pure GGNs with different invasiveness $(\mathrm{P}<0.05)$. The cut-off $\mathrm{rCT}$ values of 1,2 and nodule size for differentiating between pre-invasive and invasive pure GGNs were 293.82 [sensitivity $58.0 \%$, specificity $94.7 \%$; area under the curve (AUC) 0.783], 0.68 (sensitivity 89.5\%, specificity 58.0\%, AUC 0.742) and $1.10 \mathrm{~cm}$ (sensitivity $74.0 \%$, specificity 79.0\%, AUC 0.796), respectively. The AUCs of combining rCT values 1 and 2 with the size of nodule were 0.795 (sensitivity $62.5 \%$, specificity $89.5 \%$ ) and 0.845 (sensitivity $71.6 \%$, specificity $89.5 \%$ ) respectively. There were no significant differences in the mean CT values between pure GGNs with different levels of invasiveness and between the GGC of PSNs of MIA and IAC.

Conclusions: In patients with emphysema, the rCT values are more useful than the mean CT values for differentiating between SSNs with different invasiveness and can be valuable for patient management.

Keywords: Computed tomography (CT); emphysema; lung cancer; relative CT values (rCT values); subsolid nodules (SSN)

Submitted Nov 30, 2019. Accepted for publication Aug 13, 2020.

doi: 10.21037/qims-19-998

View this article at: http://dx.doi.org/10.21037/qims-19-998 


\section{Introduction}

Lung cancer accounts for approximately $27 \%$ of all cancer deaths (1), and adenocarcinoma is the most common histologic subtype (2). In 2011, a new international multidisciplinary classification was proposed to subdivide pulmonary adenocarcinoma into pre-invasive lesions (PL) [including atypical adenomatous hyperplasia (AAH) and adenocarcinoma in situ (AIS)], minimally invasive adenocarcinoma (MIA), and invasive adenocarcinoma (IAC), according to increasing invasiveness (3). Each stage has different management strategies, and precise diagnosis and treatment can substantially improve the survival rate of patients (4). However, early-stage adenocarcinomas with different levels of invasiveness have overlapping computed tomographic (CT) morphology and frequently appear as subsolid nodules (SSN), which are defined as hazy, nodular increased attenuation of the lung with preservation of the bronchial and vascular margins and includes pure groundglass nodule (GGN) and part-solid nodule (PSN) depending on whether there is a solid component (SC) (2). More small SSNs are being encountered with the increased use of lowdose CT screening, which poses a significant diagnostic challenge to radiologists $(5,6)$.

The CT attenuation value (CT value) of SSN is significantly associated with the extent of lepidic tumor growth at histopathologic examination (7-9). In the preinvasive stage of the lesion, tumor cells proliferate and extend along the alveolar septa, leading to thickened neoplastic septa, diminished alveoli, and pure GGN tumor on CT. As the tumor grows, the alveoli are progressively diminished and obliterated to varying degrees. The lepidic tumor evolves into a mixed lepidic and hilic tumor, resulting in a PSN on CT. Thus the CT value can be used as a discriminatory parameter for different stages of IAC (10).

Pulmonary emphysema is the morphologic enlargement of the airspaces distal to the terminal bronchiole because of dilatation or destruction of the alveoli (11). Studies have shown that patients with CT-detected emphysema are at a high risk of developing lung cancer, especially male smokers $(12,13)$. SSNs in patients with emphysema have a large air-containing space and, consequently, a low CT value as well as an unusual morphology (14). Therefore, the CT value of SSNs is influenced by emphysema (15), and the differentiating CT values of SSNs from previous studies in ordinary patients may not be applicable for patients with emphysema.

To our knowledge, the CT values of SSNs in patients with emphysema have not been investigated. This study aimed to assess whether the relative CT (rCT) values, which combine both the nodule and lung densities, can help distinguish the invasiveness of SSNs in patients with emphysema.

\section{Methods}

\section{Patients}

The institutional review board of Shanghai Chest Hospital approved this retrospective study, and informed consent was waived. A search for patients with pulmonary SSNs on chest CT from January 2014 to October 2018 was performed in the hospital's picture archiving and communication and radiology information system (PACS). The inclusion criteria were as follows: (I) SSNs $<3.0 \mathrm{~cm}$; (II) patients had high-resolution computed tomography (HRCT) target scan images of SSNs; (III) diagnostic reports included the terms "SSN" and "emphysema"; (IV) patients underwent surgical resection, and there was histological confirmation of AAH, AIS, MIA or IAC; and (V) the excised lung tissue with nodule showed emphysema histologically. Patients who had a history of chemotherapy or a biopsy for SSN were excluded. Finally, 175 consecutive patients with 187 SSNs were reviewed. AAH and AIS were classified as PL; MIA and IAC were classified as invasive.

\section{CT scanning}

CT scans were obtained with a 64-detector row scanner (Brilliance, Philips, Cleveland, OH, USA) at the end of inspiration during one breath hold. The scanning parameters of the routine CT were as follows: collimation, $0.625 \mathrm{~mm}$; pitch, 1.08; section thickness, $5.0 \mathrm{~mm}$; interval, 5.0; scan time, 5-7 s; matrix, $512 \times 512$; the field of view (FOV), $400 \mathrm{~mm} ; 120 \mathrm{kVp}$; and $250 \mathrm{~mA}$. When a lung nodule was found, a non-contrast HRCT target scan was performed with the following parameters: collimation, $0.625 \mathrm{~mm}$; pitch, 0.64; section thickness, $1.0 \mathrm{~mm}$; interval, $1.0 \mathrm{~mm}$; scan time $1-3 \mathrm{~s}$; matrix, $1,024 \times 1,024$; FOV, $180 \mathrm{~mm} ; 120 \mathrm{kVp}$; and $300 \mathrm{~mA}$.

\section{Image measurements}

CT images were reviewed individually by two radiologists (BWZ and $\mathrm{YZ}$, who had 3 and 8 years of experience in chest imaging, respectively) on the PACS. The CT values 

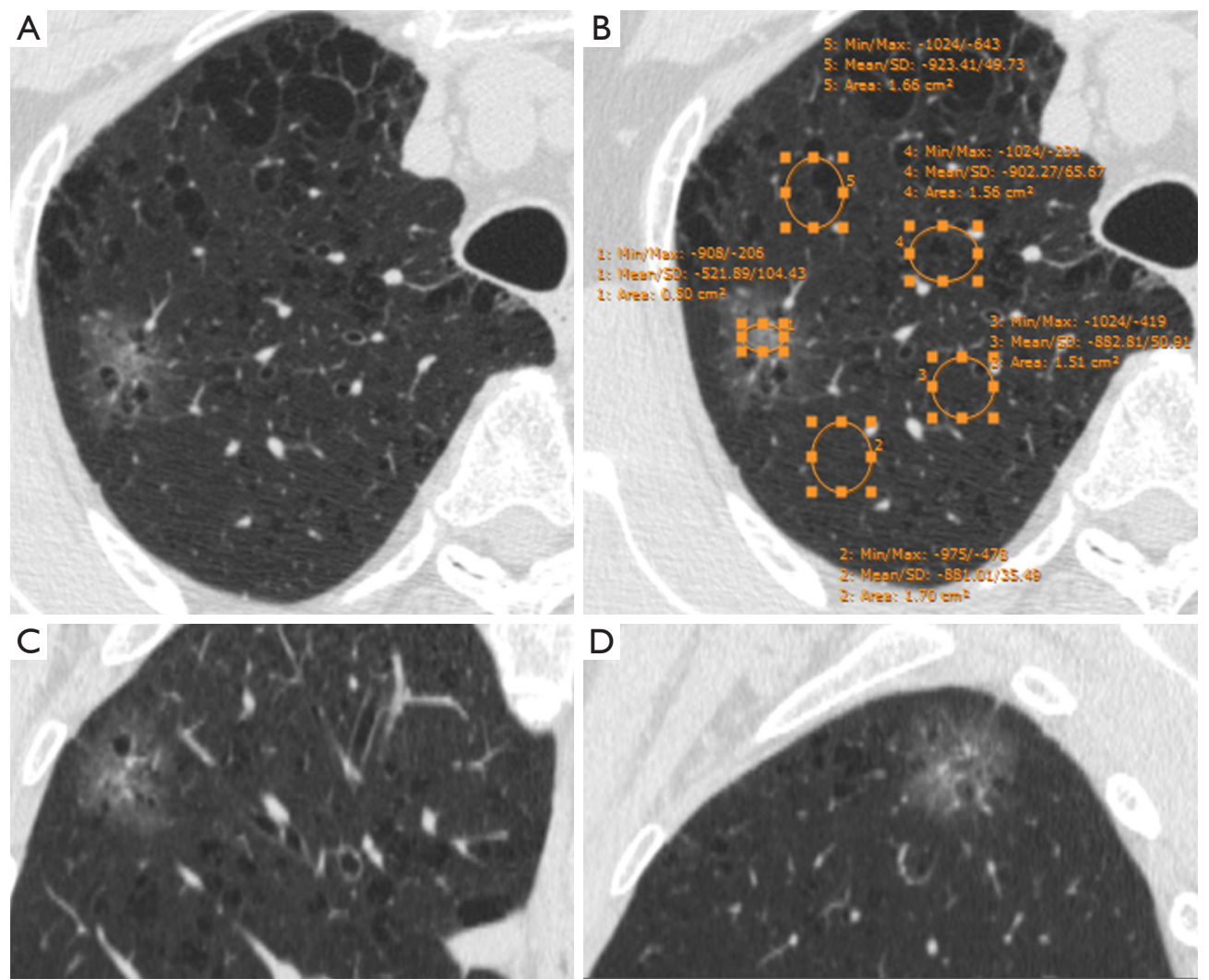

Figure 1 Lung adenocarcinoma appearing as pure GGN. (A) Axial CT image shows a pure GGN in the right upper lobe. Histology confirmed it as IAC. (B) Mean CT values of pure GGN and lung tissue are -521.89 and -897.38 HU, respectively. The relative CT values 1 and 2 are 375.48 HU and 0.58, respectively. Coronal (C) and sagittal (D) images of the nodule. CT, computed tomography; GGN, groundglass nodule; IAC, invasive adenocarcinoma.

[Hounsfield units (HU)] of the ground-glass component (GGC) and emphysematous lung were measured on a lung window setting (width, 1,450 HU; level, $-520 \mathrm{HU}$ ) by using a round or oval region of interest (ROI), covering at least one-half of the largest area of the nodule and excluding apparent vessels and bronchi (Figure 1). The CT value of the SC was measured on a mediastinal window setting (width, $350 \mathrm{HU}$; level, $40 \mathrm{HU}$ ) in the same way (Figure 2). The size of the SSN was defined as the maximal diameter in the axial section on the lung window setting, and the size of the SC of the PSN was defined as the maximal diameter in the axial section on the mediastinal window setting. All measurements of CT values were repeated three times in the nodule or four times in the nonneoplastic lung around the nodule (which histopathologically proven emphysematous lung) for each of the maximal nodule section, the adjacent upper and lower sections, respectively. The average values of 9 or 12 measurements from the first radiologist (BWZ) were selected as the representative values for the nodule and emphysematous lung. Two rCT values were adopted: 1 (nodule CT value - lung CT value) and 2 (nodule CT value/lung CT value).

\section{Statistical analysis}

Quantitative variables are described as the mean \pm standard deviation, and qualitative variables are described as frequencies (percentages). The mean CT and rCT values for different levels of SSN invasiveness were compared using a one-way analysis of variance, followed by the Student-Newman-Keuls post-test. The receiver operating characteristic (ROC) curve was performed to determine the optimal cut-off CT values and diagnostic performance for differentiating between SSNs with different levels of invasiveness. The intraclass correlation coefficient (ICC) was calculated to evaluate intraobserver variability (ICC: 


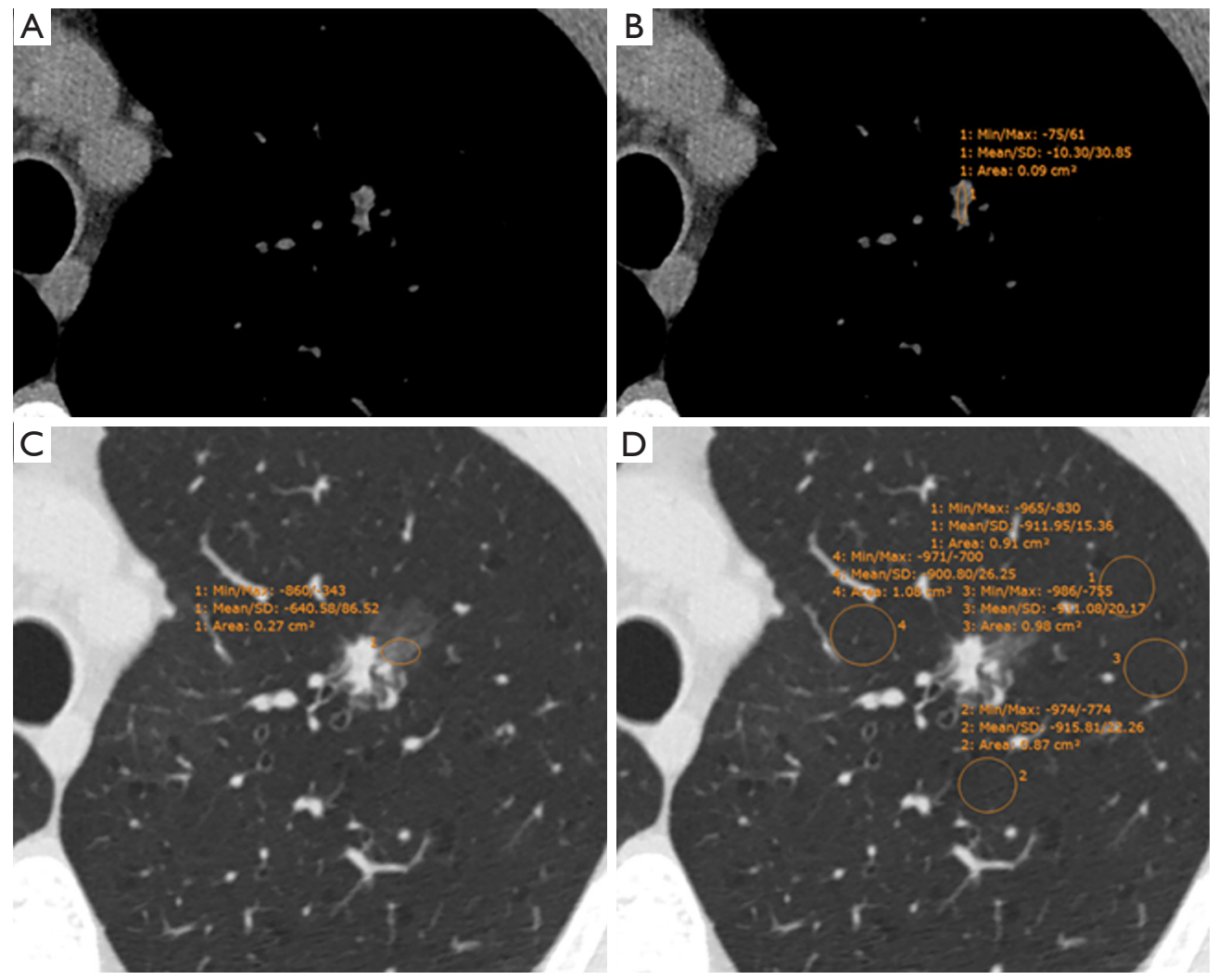

Figure 2 Lung adenocarcinoma appearing as PSN. (A) Axial CT image shows a PSN in the left upper lobe on the mediastinal window, and histology confirmed IAC. (B) The mean CT value of the SC is $-10.30 \mathrm{HU}$. (C) The mean CT value of the GGC is -640.58 HU. (D) The mean CT value of the lung is $-909.91 \mathrm{HU}$. The relative CT values 1 and 2 of the SC are $899.61 \mathrm{HU}$ and 0.01 , respectively. The relative CT values 1 and 2 of the GGC are 269.33 HU and 0.70, respectively. CT, computed tomography; GGC, ground-glass component; HU, Hounsfield units; IAC, invasive adenocarcinoma; PSN, part-solid nodule; SC, solid component.

Table 1 Features of 175 SSN patients with emphysema

\begin{tabular}{lc}
\hline Features & $\mathrm{n}$ \\
\hline Patient (cases) & 175 \\
Male & 143 \\
Female & 32 \\
Age (years) & $33-82(62.6 \pm 7.7)$ \\
SSN (n) & 187 \\
Pure GGN & 107 \\
PSN & 80 \\
CT value of lung (HU) & $-907.8 \pm 27.5$ \\
\hline
\end{tabular}

*, CT values in the lung around the SSN represent emphysematous lung (histopathologically proven). GGN, ground-glass nodule; PSN, part-solid nodule; SSN, subsolid nodule.
$0.00-0.20$, poor correlation; $0.21-0.40$, fair; $0.41-0.60$, moderate; 0.61-0.80, good; and 0.81-1.00, excellent) (16). A P value $<0.05$ was considered to be statistically significant. All statistical calculations were performed with SPSS 19.0 (Chicago, IL, USA).

\section{Results}

The 187 SSNs comprised 107 pure GGNs (7 AAHs, 12 AISs, 38 MIAs, and 50 IACs) and 80 PSNs (10 MIAs and 70 IACs); 111 (59\%) nodules were located in the right upper lobe. The features of the $175 \mathrm{SSN}$ patients with emphysema are shown in Table 1.

For the pure GGNs, the comparisons of CT values and the size of different invasive tumors are summarized in 
Table 2 Comparison of different CT values and sizes for stages of adenocarcinoma appearing as pure GGNs

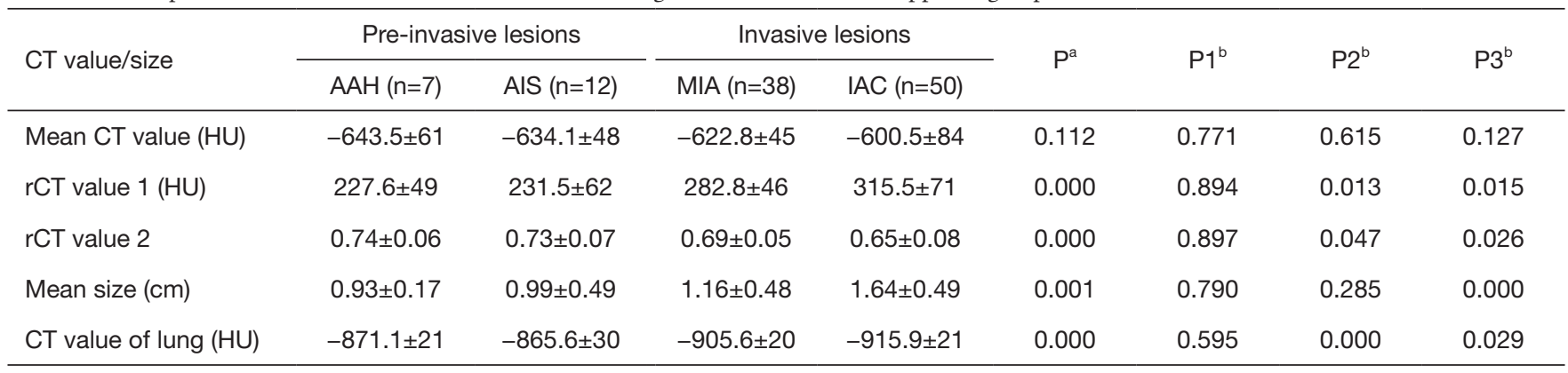

Cases of nodules shown in brackets. $\mathrm{P}$ : $\mathrm{P}$ value for pre-invasive versus invasive tumors; $\mathrm{P}$ 1: $\mathrm{P}$ value for $\mathrm{AAH}$ versus AIS; $\mathrm{P} 2$ : $\mathrm{P}$ value for AIS versus MIA; P3: P value for MIA versus IAC. ${ }^{a}$, two-tailed Student $t$-test; ${ }^{b}$, Student-Newman-Keuls post-test. AAH, atypical adenomatous hyperplasia; AIS, adenocarcinoma in situ; GGN, ground-glass nodule; IAC, invasive adenocarcinoma; MIA, minimally invasive adenocarcinoma; $r C T$, relative $\mathrm{CT}$ value.

The mean CT value (HU)

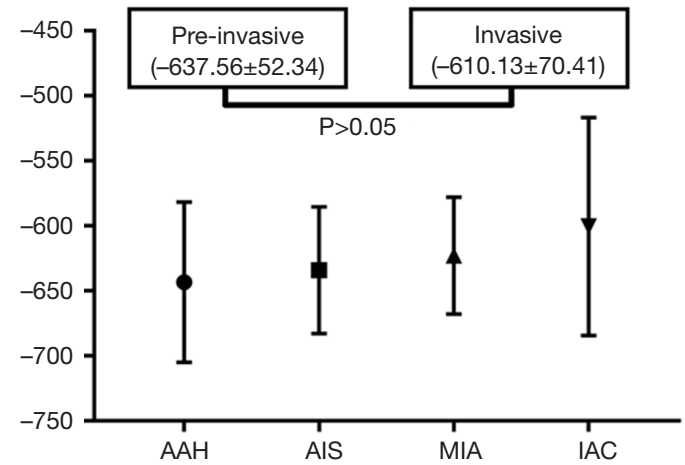

Figure 3 Plot of the mean CT value in atypical adenomatous hyperplasia (AAH), adenocarcinoma in situ (AIS), minimally invasive adenocarcinoma (MIA), and invasive adenocarcinoma (IAC) appearing as pure ground-glass nodules. CT, computed tomography; HU, Hounsfield units.

Table 2. The mean CT values in AAH, AIS, MIA, and IAC are shown in Figure 3. No significant differences in the mean CT values were observed between the different invasive pure GGNs (all $\mathrm{P}>0.05$ ). The $\mathrm{rCT}$ values of 1 and 2 in AAH, AIS, MIA, and IAC are shown in Figures 4,5. The differences in the rCT values of 1 and 2 between AIS and MIA, MIA, and IAC and between noninvasive and invasive tumors were statistically significant (all $\mathrm{P}<0.05)$. However, no significant differences in $\mathrm{rCT}$ values were observed between AAH and AIS (all $\mathrm{P}>0.05$ ). The sizes in AAH, AIS, MIA, and IAC are shown in Figure 6. The differences in size between MIA and IAC and between noninvasive and invasive tumors were statistically significant $(\mathrm{P}<0.05)$.
The relative CT value $1(\mathrm{HU})$

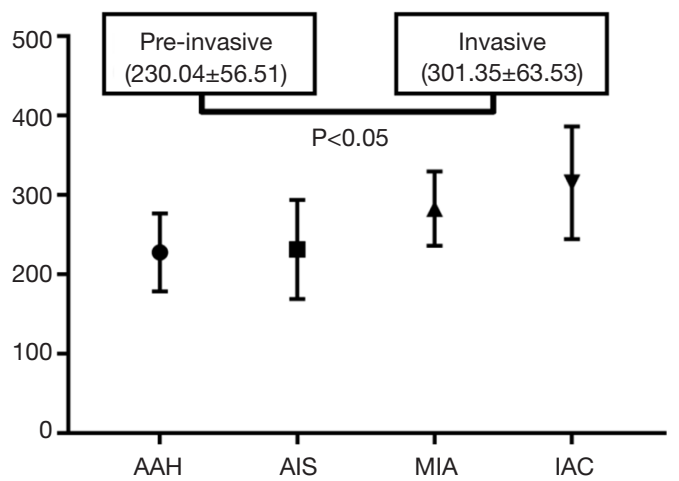

Figure 4 Plot of the relative CT value 1 in atypical adenomatous hyperplasia (AAH), adenocarcinoma in situ (AIS), minimally invasive adenocarcinoma (MIA), and invasive adenocarcinoma (IAC) appearing as pure ground-glass nodules. CT, computed tomography; HU, Hounsfield units.

The diagnostic performance of the rCT values, size of the nodules, and their combination for predicting the invasiveness of pure GGNs are shown in Table 3. The cutoff rCT values of 1 and 2 for differentiating between preinvasive and invasive pure GGNs was $293.82 \mathrm{HU}$, with a sensitivity of $58.0 \%$, the specificity of $94.7 \%$ and area under the curve (AUC) of 0.783 ; and 0.68 , with a sensitivity of $89.5 \%$, the specificity of $58.0 \%$ and AUC of 0.742 , respectively. The cut-off size for differentiating between pre-invasive and invasive pure GGNs was $1.10 \mathrm{~cm}$, with a sensitivity of $74.0 \%$, the specificity of $79.0 \%$, and AUC of 0.796 . The rCT values of 1 and 2 combined with size yielded higher AUCs of 0.795 (sensitivity $62.5 \%$, specificity 


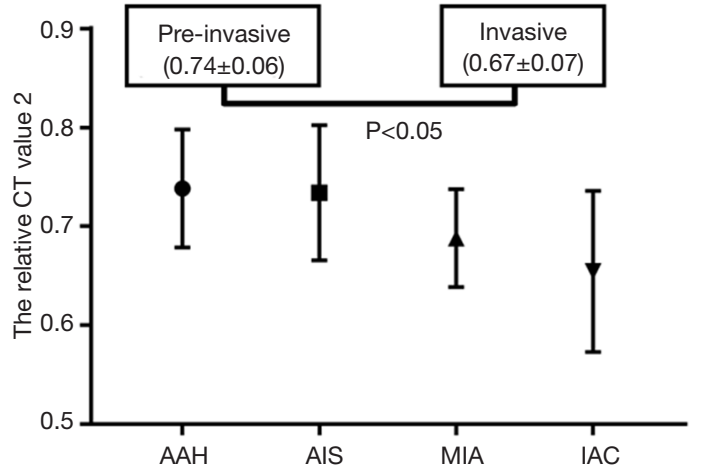

Figure 5 Plot of the relative CT value 2 in atypical adenomatous hyperplasia (AAH), adenocarcinoma in situ (AIS), minimally invasive adenocarcinoma (MIA), and invasive adenocarcinoma (IAC) appearing as pure ground-glass nodules. CT, computed tomography.

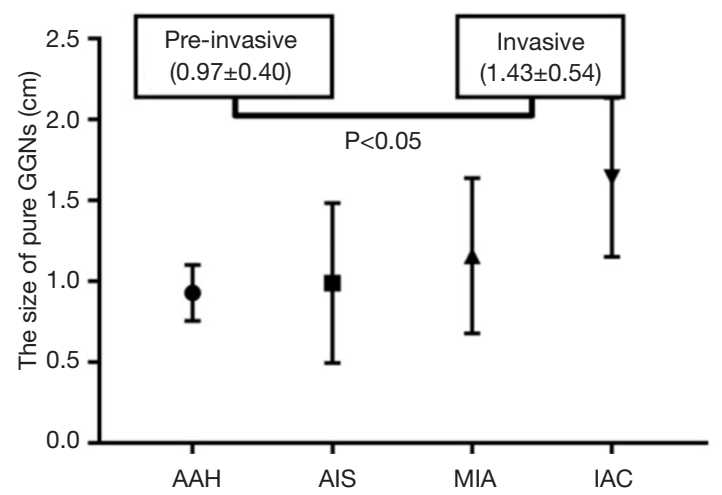

Figure 6 Plot of the size of a nodule in atypical adenomatous hyperplasia (AAH), adenocarcinoma in situ (AIS), minimally invasive adenocarcinoma (MIA), and invasive adenocarcinoma (IAC) appearing as pure ground-glass nodules (GGNs).
$89.5 \%$ ) and 0.845 (sensitivity $71.6 \%$, specificity $89.5 \%$ ), respectively.

For PSNs, the comparisons of various types of CT values between MIA and IAC are summarized in Table 4. In the GGC of the PSNs, a significant difference between MIA and IAC was shown for rCT values of 1 and 2 (all $\mathrm{P}<0.05$ ), but was not observed for the mean CT value. In the SC of PSNs, the mean CT value was $-89.79 \pm 15.34 \mathrm{HU}$ in MIA and $-49.8 \pm 61.94 \mathrm{HU}$ in IAC, with a significant difference $(\mathrm{P}<0.05)$; no significant difference between MIA and IAC was found for rCT values of 1 and 2 (all $\mathrm{P}>0.05$ ). No significant differences in all sizes of nodules were observed between MIA and IAC (all P>0.05).

ICCs for the quantitative variables are presented in Table 5. The measurement agreements were good for the CT values of pure GGN, GGC and SC of PSN, the CT value of lung, and the size of SSN, with ICCs of 0.947, $0.897,0.931,0.825$, and 0.886 , respectively.

\section{Discussion}

Management of lung cancer in patients with emphysema is challenging, and being able to predict the invasiveness of pulmonary SSNs would be an essential tool in the care of these patients (5). In our study, rCT values in patients with emphysema were better predictors than the mean CT value for the invasiveness of a tumor appearing as a pure GGN. Two rCT values for the GGC, as well as the mean CT value of the SC, were useful for differentiating between MIA and IAC in PSNs.

Many studies have shown that the mean $\mathrm{CT}$ value of nodules is useful for differentiating between various invasive lung cancers, with a higher CT value indicating

Table 3 Diagnostic performance of different rCT values, size of the nodule and their combination in distinguishing between pre-invasive and invasive adenocarcinoma appearing as pure GGNs

\begin{tabular}{lcccc}
\hline Variable & Cut-off value & Sensitivity & Specificity & AUC (95\% Cl) \\
\hline rCT value 1 $(\mathrm{HU})$ & 293.82 & $58.0 \%$ & $94.7 \%$ & $0.783(0.69-0.88)$ \\
rCT value 2 & 0.68 & $89.5 \%$ & $58.0 \%$ & $0.742(0.63-0.85)$ \\
Mean size (cm) & 1.10 & $74.0 \%$ & $79.0 \%$ & $0.796(0.68-0.90)$ \\
rCT 1-size & NA & $62.5 \%$ & $89.5 \%$ & $0.795(0.70-0.89)$ \\
rCT 2-size & NA & $71.6 \%$ & $89.5 \%$ & $0.845(0.76-0.93)$ \\
\hline
\end{tabular}

rCT 1/2-size: combining relative CT value and mean size. AUC, area under the curve; CT, computed tomography; GGN, ground-glass nodule; HU, Hounsfield units. 
Table 4 Comparison of different CT values and nodule sizes between MIA and IAC appearing as PSNs

\begin{tabular}{lccc}
\hline Variable & MIA $(\mathrm{n}=10)$ & IAC $(\mathrm{n}=70)$ & P value \\
\hline CT value of GGC $(\mathrm{HU})$ & $-630.0 \pm 34.1$ & $-612.0 \pm 66.7$ & 0.407 \\
rCT value 1 of GGC $(\mathrm{HU})$ & $282.6 \pm 11.8$ & $301.5 \pm 63.3$ & $0.029^{*}$ \\
rCT value 2 of GGC & $0.69 \pm 0.02$ & $0.67 \pm 0.07$ & $0.042^{*}$ \\
CT value of SC $(\mathrm{HU})$ & $-89.79 \pm 15.34$ & $-49.8 \pm 61.94$ & $0.047^{*}$ \\
rCT value 1 of SC $(\mathrm{HU})$ & $822.81 \pm 48.07$ & $863.67 \pm 83.4$ & 0.135 \\
rCT value 2 of SC & $0.10 \pm 0.02$ & $0.06 \pm 0.07$ & 0.067 \\
Size of PSN $(\mathrm{cm})$ & $1.88 \pm 0.60$ & $1.95 \pm 0.62$ & 0.736 \\
Size of SC $(\mathrm{cm})$ & $0.84 \pm 0.43$ & $0.90 \pm 0.49$ & 0.711 \\
SC/GGC ratio & $0.44 \pm 0.16$ & $0.45 \pm 0.17$ & 0.854 \\
CT value of lung $(\mathrm{HU})$ & $-912.6 \pm 34$ & $-913.5 \pm 26$ & 0.923 \\
\hline
\end{tabular}

Cases of nodules shown in brackets. *, P<0.05. CT, computed tomography; GGC, ground-glass component; HU, Hounsfield units; PSN, part-solid nodule; SC, solid component.

Table 5 Intraclass correlation coefficient (ICC) between two observers

\begin{tabular}{llc}
\hline Variable & ICC & $95 \% \mathrm{Cl}$ \\
\hline Mean CT value of pure GGN & 0.947 & $0.923-0.964$ \\
Mean CT value of GGC in PSN & 0.897 & $0.843-0.932$ \\
Mean CT value of SC in PSN & 0.931 & $0.894-0.955$ \\
Mean CT value of lung & 0.825 & $0.773-0.866$ \\
Nodule size & 0.886 & $0.857-0.909$
\end{tabular}

$\mathrm{Cl}$, confidence interval; CT, computed tomography; GGC, ground-class component; GGN, ground-glass nodule; PSN, part-solid nodule; SC, solid component.

a greater possibility for malignancy (17-19). Ikeda et al. demonstrated that a CT cut-off value of $-584 \mathrm{HU}$ was useful for differentiating between AAH and AIS, and $-472 \mathrm{HU}$ was effective for differentiating between AIS and invasive adenocarcinoma (20). Another study by Eguchi et al. (21) found that pure GGNs grew slowly during longterm follow-up and that the mean CT value was useful for predicting the growth of pure GGNs. Their cut-off value was set at $-670 \mathrm{HU}$. However, these previous studies did not mention whether patients with SSN had emphysema (17-21). In the present study of patients with emphysema, the mean CT value was not significantly different between pre-invasive and invasive SSNs. One study has verified a strong negative association between CT attenuation and retained air space in tumors (22).

The mean CT value is the average attenuation of all pixels in the ROI and a comprehensive voxels representative of the alveolar walls, septa, air, and interstitial tissue. The mean CT value is approximately $-850 \mathrm{HU}$ according to inspiratory status in normal lungs (23), ranges from -900 to $-1,024 \mathrm{HU}$ in emphysematous areas (24), and was $-907.82 \pm 27.53 \mathrm{HU}$ in this study's 175 emphysematous patients with SSN. Nonneoplastic lung was also emphysematous on histopathology, and according to the CT values, the present study's patients had mild emphysema. The mean CT value of SSN mainly depends on the number of tumor cells, the retained alveolar air, and collapsed interstitial stroma mixed in a mosaic pattern. Between the same invasive SSNs of ordinary and emphysematous lung cancer patients, there should not be a significant difference in the number of tumor cells. However, there should be a significant difference in the amount of alveolar air, 
according to common histopathologic sense. Differences in the CT values of SSNs among the various invasive types may be influenced by the amount of alveolar air in the lung tissue. More air was retained within the emphysematous alveoli of invasive SSNs, which could compromise the attenuation difference among the various invasive SSNs, and consequently, the differentiating performance of the mean CT values, as was observed in this study. Therefore, we developed two rCT values to eliminate the negative effect of emphysema and to quantify the number of tumor cells and their proportion with alveolar air in SSNs. The rCT value of 1 represented the number of tumor cells, which was positively correlated with the invasiveness of SSN. The rCT value of 2 reflected the relative density of the SSN.

In pure GGNs, our study found that $\mathrm{rCT}$ values of 1 and 2 were better markers than the mean CT value for differentiating between AIS and MIA, between MIA and IAC and between pre-invasive and invasive tumors. The optimal cut-off values of the invasiveness of pure GGNs were $>293.82 \mathrm{HU}$ and $<0.68$, with a sensitivity of $58.0 \%$ and $89.5 \%$, the specificity of $94.7 \%$ and $58.0 \%$, and AUC of 0.783 and 0.742 , respectively. The rCT value of 1 was the better marker of the two rCT values, with higher sensitivity and AUC because it quantified the amount of tumor tissue by excluding the negative effect of the background emphysematous lung. Zhao et al. (25) described the "relative density" as the lung CT value divided by the nodule CT value. They found that the "relative density" was higher in the IAC than in the pre-invasive/MIA group, and a "relative density" $>1.60$ can increase the risk of IAC in pure GGNs by 4.4-fold. They thought that the "relative density" could eliminate the confounding of the contrast agent and reduce the effect of respiratory status. Also, we found no differences in the two rCT values between AAH and AIS, which is likely due to the little difference between AAH and AIS in histopathologic appearance (26). The diagnosis of AIS cannot be firmly established without a histological sampling of the entire tumor (3).

In PSNs, our study showed that the rCT values for the GGC and the mean CT value of the SC were significantly different in MIA and IAC. No significant differences were found for the mean CT of the GGC, or the two rCT values of the SC, between MIA and IAC. The rCT values were more sensitive markers for the attenuation difference of the GGC, whereas the mean CT value better reflected the attenuation difference of the SC between MIA and IAC. The results were following the findings of previous studies that showed that the mean CT value of the SC of the PSNs was significantly higher in MIA than in AAH-AIS and in IAC than in AIS-MIA $(8,27)$. A reasonable explanation is that SC is usually considered to represent the invasive component of the tumor (28). Lim et al. (29) showed that the size of the SC of PSNs on CT was significantly smaller than the size of the invasive component on pathology in patients with emphysema. That result means that the GGC of PSNs on CT could reflect an invasive lesion. Due to emphysema in our study, however, the differences in the absolute CT values between MIA and IAC in the GGC were too small to be identified by mean CT values, and the SC of PSNs reflected the absence of retained alveolar air and thus was not influenced by emphysema. Some studies suggest that a vacuole is associated with more IACs and is found more frequently in IAC than in AAH and AIS (30,31).

A large number of studies have confirmed that the size of SSN is an independent predictor for differentiating benign from malignant nodules (32-34). Size has played a crucial role in SSN management of several lung cancer screening trials (5). In our study, the size of pure GGN was significantly different between pre-invasive and IACs, but there were no significant differences in the size of pure GGNs between AIS and MIA and in the size of PSNs and SCs between MIA and IAC. These differences are also likely due to the smaller size differences existing between different invasive tumors in patients with emphysema, as mentioned before (29). Also, it is challenging to differentiate hyperplastic tissue between dilated alveoli from real invasive components on CT (35). However, by combining the rCT value 2 with size, greater AUCs were achieved for differentiating invasive from preIACs than by the size only (0.845 vs. 0.796).

This study had several limitations. First, it was retrospective, and only histologically proven SSN patients with emphysema were included. Therefore, selection bias was inevitable. Second, the method for assessing the CT attenuation values was fundamental $(2 \mathrm{D}$, round ROI without segmentation), though efforts were made to avoid measurement bias, including the average of multiple measurements from multiple ROIs and sections, and two independent radiologists. Third, although emphysema in the patients with nodules was proved by both CT and histopathology and was found in the same lung segment, no point-to-point correlation between CT and histopathology was performed. 


\section{Conclusions}

In patients with emphysema, $\mathrm{rCT}$ values are more useful than the mean CT value for differentiating between SSNs with different invasiveness and can be valuable for patient management. A combination of rCT value 2 and size can achieve better performance than the $\mathrm{rCT}$ value or size alone.

\section{Acknowledgments}

Funding: None.

\section{Footnote}

Conflicts of Interest: All authors have completed the ICMJE uniform disclosure form (available at http://dx.doi. org/10.21037/qims-19-998). The authors have no conflicts of interest to declare.

Ethical Statement: The institutional review board of Shanghai Chest Hospital approved this retrospective study, and informed consent was waived.

Open Access Statement: This is an Open Access article distributed in accordance with the Creative Commons Attribution-NonCommercial-NoDerivs 4.0 International License (CC BY-NC-ND 4.0), which permits the noncommercial replication and distribution of the article with the strict proviso that no changes or edits are made and the original work is properly cited (including links to both the formal publication through the relevant DOI and the license). See: https://creativecommons.org/licenses/by-nc-nd/4.0/.

\section{References}

1. American Cancer Society. Cancer facts \& figures 2015. Atlanta: American Cancer Society; 2015.

2. Hansell DM, Bankier AA, MacMahon H, McLoud TC, Muller NL, Remy J. Fleischner Society: glossary of terms for thoracic imaging. Radiology 2008;246:697-722.

3. Travis WD, Brambilla E, Noguchi M, Nicholson AG, Geisinger KR, Yatabe Y, Beer DG, Powell CA, Riely GJ, Van Schil PE, Garg K, Austin JH, Asamura H, Rusch VW, Hirsch FR, Scagliotti G, Mitsudomi T, Huber RM, Ishikawa Y, Jett J, Sanchez-Cespedes M, Sculier JP, Takahashi T, Tsuboi M, Vansteenkiste J, Wistuba I, Yang PC, Aberle D, Brambilla C, Flieder D, Franklin W,
Gazdar A, Gould M, Hasleton P, Henderson D, Johnson B, Johnson D, Kerr K, Kuriyama K, Lee JS, Miller VA, Petersen I, Roggli V, Rosell R, Saijo N, Thunnissen E, Tsao M, Yankelewitz D. International association for the study of lung cancer/American thoracic society/European respiratory society international multidisciplinary classification of lung adenocarcinoma. J Thorac Oncol 2011;6:244-85.

4. Wood DE. National comprehensive cancer network (NCCN) clinical practice guidelines for lung cancer screening. Thorac Surg Clin 2015;25:185-97.

5. MacMahon H, Naidich DP, Goo JM, Lee KS, Leung ANC, Mayo JR, Mehta AC, Ohno Y, Powell CA, Prokop M, Rubin GD, Schaefer-Prokop CM, Travis WD, Van Schil PE, Bankier AA. Guidelines for management of incidental pulmonary nodules detected on CT images: from the Fleischner Society 2017. Radiology 2017;284:228-43.

6. Hammer MM, Palazzo LL, Kong CY, Hunsaker AR. Cancer risk in subsolid nodules in the national lung screening trial. Radiology 2019;293:441-8.

7. Lee HY, Choi YL, Lee KS, Han J, Zo JI, Shim YM, Moon JW. Pure ground-glass opacity neoplastic lung nodules: histopathology, imaging, and management. AJR Am J Roentgenol 2014;202:W224-33.

8. Li Q, Fan L, Cao E, Li Q, Gu Y, Liu S. Quantitative CT analysis of pulmonary pure ground-glass nodule predicts histological invasiveness. Eur J Radiol 2017;89:67-71.

9. Zhang Y, Shen Y, Qiang J, Ye J, Zhang J, Zhao R. HRCT features distinguishing pre-invasive from invasive pulmonary adenocarcinomas appearing as ground-glass nodules. Eur Radiol 2016;26:2921-8.

10. Eguchi T, Yoshizawa A, Kawakami S, Kumeda H, Umesaki T, Agatsuma H, Sakaizawa T, Tominaga Y, Toishi M, Hashizume M, Shiina T, Yoshida K, Asaka S, Matsushita M, Koizumi T. Tumor size and computed tomography attenuation of pulmonary pure ground-glass nodules are useful for predicting pathological invasiveness. PLoS One 2014;9:e97867.

11. The definition of emphysema. Report of a National Heart, Lung, and Blood Institute, Division of Lung Diseases workshop. Am Rev Respir Dis 1985;132:182-5.

12. Smith BM, Pinto L, Ezer N, Sverzellati N, Muro S, Schwartzman K. Emphysema detected on computed tomography and risk of lung cancer: a systematic review and meta-analysis. Lung Cancer 2012;77:58-63.

13. Usui K, Tanai C, Tanaka $Y$, Noda H, Ishihara T. 
The prevalence of pulmonary fibrosis combined with emphysema in patients with lung cancer. Respirology 2011;16:326-31.

14. Matsuoka S, Kurihara Y, Yagihashi K, Niimi H, Nakajima Y. Peripheral solitary pulmonary nodule: CT findings in patients with pulmonary emphysema. Radiology 2005;235:266-73.

15. Kim EY, Seo JB, Lee HJ, Kim N, Lee E, Lee SM, Oh SY, Hwang HJ, Oh YM, Lee SD. Detailed analysis of the density change on chest $\mathrm{CT}$ of COPD using non-rigid registration of inspiration/expiration CT scans. Eur Radiol 2015;25:541-9.

16. Cicchetti DV, Sparrow SA. Developing criteria for establishing interrater reliability of specific items: applications to assessment of adaptive behavior. Am J Ment Defic 1981;86:127-37.

17. Yanagawa M, Kuriyama K, Kunitomi $Y$, Tomiyama N, Honda O, Sumikawa H, Inoue A, Mihara N, Yoshida S, Johkoh T, Nakamura H. One-dimensional quantitative evaluation of peripheral lung adenocarcinoma with or without ground-glass opacity on thin-section CT images using profile curves. Br J Radiol 2009;82:532-40.

18. Tamura M, Shimizu Y, Yamamoto T, Yoshikawa J, Hashizume Y. Predictive value of one-dimensional mean computed tomography value of ground-glass opacity on high- resolution images for the possibility of future change. J Thorac Oncol 2014;9:469-72.

19. Kitami A, Kamio Y, Hayashi S, Suzuki K, Uematsu S, Gen R, Suzuki T, Kadokura M. One-dimensional mean computed tomography value evaluation of groundglass opacity on high-resolution images. Gen Thorac Cardiovasc Surg 2012;60:425-30.

20. Ikeda K, Awai K, Mori T, Kawanaka K, Yamashita Y, Nomori H. Differential diagnosis of ground-glass opacity nodules: CT number analysis by three-dimensional computerized quantification. Chest 2007;132:984-90.

21. Eguchi T, Kondo R, Kawakami S, Matsushita M, Yoshizawa A, Hara D, Matsuoka S, Takeda T, Miura K, Agatsuma H, Sakaizawa T, Tominaga Y, Saito G, Toishi M, Hamanaka K, Hashizume M, Shiina T, Amano J, Koizumi T, Yoshida K. Computed tomography attenuation predicts the growth of pure ground-glass nodules. Lung Cancer 2014;84:242-7.

22. Yang ZG, Sone S, Takashima S, Li F, Honda T, Maruyama Y, Hasegawa M, Kawakami S. High-resolution CT analysis of small peripheral lung adenocarcinomas revealed on screening helical CT. AJR Am J Roentgenol 2001;176:1399-407.
23. Fernandes L, Fernandes Y, Mesquita AM. Quantitative computed tomography imaging in chronic obstructive pulmonary disease. Lung India 2016;33:646-52.

24. Mao JT, Goldin JG, Dermand J, Ibrahim G, Brown MS, Emerick A, McNitt-Gray MF, Gjertson DW, Estrada F, Tashkin DP, Roth MD. A pilot study of all-trans-retinoic acid for the treatment of human emphysema. Am J Respir Crit Care Med 2002;165:718-23.

25. Zhao Q, Wang J, Yang L, Xue L, Lu W. CT diagnosis of pleural and stromal invasion in malignant subpleural pure ground-glass nodules: an exploratory study. Eur Radiol 2019;29:279-86.

26. Sakurai H, Maeshima A, Watanabe S, Suzuki K, Tsuchiya R, Maeshima AM, Matsuno Y, Asamura H. Grade of stromal invasion in small adenocarcinoma of the lung: histopathological minimal invasion and prognosis. Am J Surg Pathol 2004;28:198-206.

27. Zhang Y, Qiang J, Ye J, Ye X, Zhang J. High resolution $\mathrm{CT}$ in differentiating minimally invasive component in early lung adenocarcinoma. Lung Cancer 2014;84:236-41.

28. Park CM, Goo JM, Lee HJ, Chun EJ, Im JG. Nodular ground-glass opacity at thin-section CT: histologic correlation and evaluation of change at follow-up. Radiographics 2007;27:391-408.

29. Lim JK, Shin KM, Lee SY, Lee H, Hahm MH, Lee J, Kim CH, Cha SI, Jeong JY. Can emphysema influence size discrepancy between radiologic and pathologic size measurement in subsolid lung adenocarcinomas? Thoracic Cancer 2019;10:1919-27.

30. Kim TJ, Goo JM, Lee KW, Park CM, Lee HJ. Clinical, pathological and thin-section CT features of persistent multiple ground-glass opacity nodules: comparison with solitary ground-glass opacity nodule. Lung cancer 2009;64:171-8.

31. Koo CW, Miller WT, Kucharczuk JC. Focal groundglass opacities in non-small cell lung carcinoma resection patients. Eur J Radiol 2012;81:139-45.

32. Parmar C, Leijenaar RT, Grossmann P, Rios Velazquez E, Bussink J, Rietveld D, Rietbergen MM, Haibe-Kains B, Lambin P, Aerts HJ. Radiomic feature clusters and prognostic signatures specific for Lung and Head \& Neck cancer. Sci Rep 2015;5:11044.

33. Coroller TP, Grossmann P, Hou Y, Rios Velazquez E, Leijenaar RT, Hermann G, Lambin P, Haibe-Kains B, Mak RH, Aerts HJ. CT-based radiomic signature predicts distant metastasis in lung adenocarcinoma. Radiother Oncol 2015;114:345-50.

34. Gillies RJ, Kinahan PE, Hricak H. Radiomics: images 
are more than pictures, they are data. Radiology 2016;278:563-77.

35. Lang MR, Fiaux GW, Gillooly M, Stewart JA, Hulmes
DJ, Lamb D. Collagen content of alveolar wall tissue in emphysematous and non-emphysematous lungs. Thorax 1994;49:319-26.
Cite this article as: Zhang BW, Zhang Y, Ye JD, Qiang JW. Use of relative CT values to evaluate the invasiveness of pulmonary subsolid nodules in patients with emphysema. Quant Imaging Med Surg 2021;11(1):204-214. doi: 10.21037/qims-19998 